\title{
Wavelength Dependent Anisotropic Photo Sensing Activity of Zirconium Trisulfide Crystal
}

\section{Anand Chunilal Patel ( $\nabla$ acpatel1996@gmail.com )}

Sardar Patel University https://orcid.org/0000-0001-7066-4273

\section{Kunjal Patel}

Sardar Patel University

Chaitanya Limberkar

Sardar Patel University

K. D. Patel

Sardar Patel University

G. K. Solanki

Sardar Patel University

V. M. Pathak

Sardar Patel University

\section{Research Article}

Keywords: Crystal growth, Device fabrication, Anisotropy, Photoresponse, Responsivity

Posted Date: February 23rd, 2021

DOI: https://doi.org/10.21203/rs.3.rs-226790/v1

License: (c) (i) This work is licensed under a Creative Commons Attribution 4.0 International License.

Read Full License 


\section{Abstract}

According to the performance requirements, either bulk or nanocrystalline form of the material can be used for different types of device applications. In the present study, zirconium triselenide bulk crystals were grown by direct vapour transport technique. The as-grown crystals and powder have been examined under Carl Zeiss optical microscope and scanning electron microscope for morphological studies which revealed the evolution of crystalline phases of the material by the layered kind of growth mechanism. The energy dispersive analysis of X-ray (EDAX) characterization confirms that no impurity is found in the resulting product except the desired material. To study the effect of different wavelength sources (Blue$470 \mathrm{~nm}$, Green-540 nm, Red-670 nm) on bulk zirconium trisulfide photodetectors, pulsed photo response experiment was carried out. The anisotropic behaviour is also revealed using the same sources. Various device parameters like responsivity, sensitivity, detectivity and external quantum efficiency (EQE\%) were calculated. The highest responsivity and detectivity of $81.7 \mu \mathrm{A} / \mathrm{W}$ and $3.56 \times 10^{07}$ Jones were achieved for blue $(470 \mathrm{~nm})$ light source respectively.

\section{Introduction}

Two-dimensional (2D)-layered materials have emerged as a new class of materials with unusual optical, electrical, mechanical and thermal properties. Owing to their unique properties, they attracted researcher's attention for applications in energy conversion, flexible electronics and information technology fields ${ }^{1-3}$. Transition Metal Tarichalcogenides (TMTCs) of layered group-IV with the chemical formula of $\mathrm{MX}_{3}$, e.g., $\mathrm{M}=\mathrm{Zr}, \mathrm{Ti}$ and $\mathrm{X}=\mathrm{S}$, Se, Te, are known to be anisotropic $2 \mathrm{D}$ systems ${ }^{4,5}$. These trichalcogenides are often synthesized in thin fibrous ribbons shape and offer several interesting phenomena originating from their large structural in-plane anisotropy as individual $\mathrm{MX}_{3}$ layers are made out of moderately interacting 1Dlike chain structures ${ }^{6-9}$. Among these materials, particularly, transition metal trisulfides (TMTS) are receiving attention of researchers because of the number of inherent properties. As the groups $(S-S)^{2-}$ has the capability of reversibly accepting a pair of electrons ${ }^{10}$, these species are studied as cathode materials in chemical current sources ${ }^{11}$, including $\mathrm{ZrS}_{3}$ for primary thermal Li-battery as well as semiconductor $\mathrm{TiS}_{3}{ }^{12}$. Some transition metal polysulfides, in particular semiconducting MS3, are of interest as catalysts in the production of oxygen $(\mathrm{ZrS} 3)^{13}$ or as gas sensors $(\mathrm{TaS} 3)^{14}$. Zirconium trisulfide $\left(\mathrm{ZrS}_{3}\right)$ attracted interest owing to theoretically calculated $1.79 \mathrm{eV}$ bandgap value for bulk $\mathrm{ZrS}_{3}{ }^{15}$. Recently, light sensors based on $\mathrm{ZrS}_{3}$ material were fabricated using the nanoribbons and nanobelts forms and tested under various illumination sources like LASER ${ }^{16}$, white light ${ }^{17}$, Ultra violate to NIR ${ }^{18}$ and was reported. These devices showed good responsivity towards respective illuminations with minimum external bias. Despite these recent researches, this light sensing capacity of the bulk crystalline material has not yet been studied. Also, the light sensing activity of both in-plane and out-plane symmetries is not yet revealed. In this present study, the growth process, characterization and application of $\mathrm{ZrS}_{3}$ material in its bulk crystalline form is demonstrated. 


\section{Experimental}

\subsection{Growth of $\mathrm{ZrS}_{3}$ crystal}

The bulk $\mathrm{ZrS}_{3}$ crystals were grown by direct vapour transport technique. Initially, the inner surface of a quartz ampoule having dimensions $24 \mathrm{~cm}$ in length, $2.2 \mathrm{~cm}$ inner diameter and $2.4 \mathrm{~cm}$ outer diameter was cleaned with sulfuric acid, hydrochloric acid and simple water periodically one by one. The bottom part of the ampoule was etched by evaporating hydrofluoric acid at $100{ }^{\circ} \mathrm{C}$ temperature, to create enough rough surface that provide nucleation points. Further the ampoule was cleaned with simple water and acetone and set to dry in oven at $200^{\circ} \mathrm{C}$. This highly cleaned ampoule was then filled with pure zirconium (99.99\%) and sulfur (99.99\%) powder with stoichiometric proportions and sealed at a pressure better than $10^{-5}$ Torr. This sealed ampoule was then inserted into a high temperature dual zone horizontal furnace. The ampoule was set such as the half part is situated in higher temperature zone and other half part in lower temperature zone. The temperature of the furnace was initially raised up to $850{ }^{\circ} \mathrm{C}$ for $40 \mathrm{hrs}$, the reaction time was set for $60 \mathrm{hrs}$ at maximum temperature and in last stage the furnace was cool down to room temperature in $80 \mathrm{hrs}$ with slow temperature decrement. The temperature difference between the two zones was maintained $50{ }^{\circ} \mathrm{C}$ throughout the cycle. The process yielded needle shaped thin flakes with shiny metallic appearance.

\subsection{Characterization}

The as grown compound was analysed quantitatively by performing energy dispersive analysis of X-ray analysis with elemental mapping (Fig. 2). The calculated and observed values of weight\% of the constituent elements are mentioned in Table. 1. The scanning electron microscopy of as grown $\mathrm{ZrS}_{3}$ compound was carried out which is shown in Fig. $3(c, d)$. Both the EDAX and SEM analysis were performed using a single instrument named FEG-SEM-450. The grown crystal was analysed under the Carl-Zeiss optical microscope to study the nature of the surface of the as grown $\mathrm{ZrS}_{3}$ crystal (Fig. 3 (a, b)).

Further, the grown single crystal was characterized for the study of the photo sensing activity. To study the ability and efficiency of $\mathrm{ZrS}_{3}$ single crystal, it was analysed under the Carl-Zeiss optical microscope the smooth surface essential for the fabrication of good quality photo detector can be seen here. To fabricate the photo detector, a shiny needle shaped $\mathrm{ZrS}_{3}$ crystal having area of $0.04 \mathrm{~cm}^{2}$ was cleaved using scotch tape to get fresh and clean surface. This crystal was then attached on a piece of mica sheet $\left(1 \times 1 \mathrm{~cm}^{2}\right)$. Two copper wires were attached at two ends of the crystal to get in plane symmetry (parallel to the basal plane) and one is made at the bottom side of the crystal to get out of plane symmetry (conduction perpendicular to the basal plane) using conductive silver paste (Fig. 1). This mica sheet was further attached on a PCB which was connected to Keithly 4200 SCS. The schematic diagram of prepared device and connections is shown in Fig. 1. Pulse photo response experiment was carried out to study the switching behaviour of $\mathrm{ZrS}_{3}$ crystal towards three different wavelength sources (Blue- $670 \mathrm{~nm}$, Green-540, Red-470 nm) for in plane and out plan contact symmetries. 


\section{Results And Discussion}

\subsection{Material characterization}

In the growth process, the temperature was raised gradually in the first cycle to turn the constituent elements into vapour form from pure metal powder steadily. The second cycle was set for a constant temperature value so that these vaporised materials react with each other properly. In the last cycle, the furnace was cooled down to the room temperature such that material get condensed in crystallin form based on the nucleation points created at the curvy part of the ampoule where etching was done. At the end fine shiny metallic crystals were collected. The elemental investigation of $\mathrm{ZrS}_{3}$ was carried out using EDAX. Table 1 shows the calculated and observed stoichiometric proportion of the constituent elements i.e. zirconium and sulphur. Fig. 2 shows uniform distribution of $\mathrm{Zr}$ and $\mathrm{S}$ throughout the area covered by the as grown compound crystal. The observed values are near to the calculated values and the final product is zirconium rich material as per the EDAX data.

Table 1. Standard and observed weight percentage of elements obtained from EDAX

\begin{tabular}{|c|c|c|c|c|}
\hline \multirow[t]{2}{*}{ Sample } & \multicolumn{2}{|c|}{ Calculated Weight \% } & \multicolumn{2}{|c|}{ Observed Weight \% } \\
\hline & $\mathrm{Zr}$ & $\mathrm{S}$ & $\mathrm{Zr}$ & $\mathrm{S}$ \\
\hline $\mathrm{ZrS}_{3}$ & 48.67 & 51.33 & 50.05 & 49.95 \\
\hline
\end{tabular}

The few fresh as grown crystals were then selected for the study under the optical microscope. The optical image of the as grown $\mathrm{ZrS}_{3}$ crystals are shown in Fig. $\mathbf{3}(\mathbf{a}, \mathbf{b})$ having dimensions in few micrometres is seen and the layers produced during the growth process are clearly been seen. In Fig. 3 (a), the edge of a crystal is shown in which the staking of layers is observed. In Fig. $\mathbf{3}$ (b) shows the top view of the crystal in which layers are produced with pointed end. The vertically staked layers depicts that the grown material has multilayer structure which is suitable for the anisotropic studies ${ }^{19}$. Fig. $\mathbf{3}$ (c) and (d) shows the SEM image of as grown compound. The grown compound contains strips having length and width in micrometre range. These strips are nothing but well grown micro crystals of $\mathrm{ZrS}_{3}$ materials. In Fig. (d), a monoclinic shaped object is found. In many previously published research articles, it is claimed that $\mathrm{ZrS}_{3}$ has monoclinic crystal structure. So, this object found in the SEM image indicates in a confirmative way that the grown material has monoclinic structure.

\subsection{Current - time characteristics}

$\operatorname{TMTC}^{20,21,22}$ and TMDC $23,24,25$ materials are layered materials which lead to the orientation dependent in material properties in different directions. Due to the staking of layers, these TMC materials provides anisotropy ${ }^{16}$ perpendicular to the basal plan. Many researchers demonstrated this anisotropy fact theoretically as well as experimentally. Anisotropic thermoelectric properties of SnSe and SnS was studied by Guo et al. using First principles method ${ }^{26}$. C. H. Ho et al. and Chauhan et al. showed strong in- 
plane anisotropy in electrical properties in pristine and doped $\operatorname{ReX}_{2}(X=$ Se and $S)$ TMDCs respectively ${ }^{27,19}$. Patel K. et al. worked anisotropic variations in electrical properties of $\mathrm{ZrS}_{x} \mathrm{Se}_{3-\mathrm{x}}$ series $^{28}$. Owing to the previously published articles for anisotropy in layered materials, $\mathrm{ZrS}_{3}$ crystal is also expected to display strong anisotropy. We, therefore, investigated the anisotropic photo response of $\mathrm{ZrS}_{3}$ crystal. To study the anisotropy of grown $\mathrm{ZrS}_{3}$ crystal in terms of current-voltage (I- $\left.\mathrm{V}\right)$ and current-time (It) characteristics, two types of contacts were taken: (1) conduction along the basal plane ( to c-axis) and (2) conduction perpendicular to the basal plane ( to c-axis) as shown in Fig. 1. The corresponding (I-V) curves for both configurations are shown in Fig. 4 (a) and (c) respectively. The I-V characteristics of $\mathrm{ZrS}_{3}$ crystal was carried out under the dark and three monochromatic illumination sources i. e. blue $(470 \mathrm{~nm})$, green $(540 \mathrm{~nm})$ and red $(670 \mathrm{~nm})$ LEDs having power $100 \mathrm{~mW} / \mathrm{cm}^{2}$ at $1 \mathrm{~V}$ external bias using Keithley 4200 SCS. For that, copper electrodes were attached on the crystals with the help of conductive silver paste. The I-V characteristics of the $\mathrm{ZrS}_{3}$ crystal under dark and illumination condition are shown in Fig. 4 (a) and (c) for both types of contact configurations. It can be inferred from the graph that $\mathrm{ZrS}_{3}$ device show good ohmic nature for similar contacts. In both contact configurations, the current increases upon illuminating the device by different lights, indicating good photo-response of the material. Besides, it is observed that the current along the direction parallel to the basal plan is higher than perpendicular to the basal plan. The dark current along the parallel direction is $447.7 \mathrm{nA}$, larger than $61.15 \mathrm{nA}$ along the perpendicular direction. The device was illuminated by the blue $(470 \mathrm{~nm})$ light, the current rises to 0.448 $\mu \mathrm{A}$ in contacts to c-axis whereas the current rises to $0.061 \mu \mathrm{A}$ in contacts to c-axis. These values of current inferred the strong anisotropy of $\mathrm{ZrS}_{3}$. This anisotropy is also demonstrated well in I-t plots shown in Fig. 4 (b) and (d). Chauhan P. et al. described effect on the current flow mechanism in these kinds of two symmetric ( to c-axis) and anti-symmetric contact( to c-axis) configurations by demonstrating a model ${ }^{19}$. In consideration of symmetric contact configuration, few upper layers of the multilayer crystal are affected by the light source according to the depth of absorption of incident photons and bias voltage. These are the only layers which may get involved in the dominant conduction. These layers have zirconium and sulphur atoms bonded with covalent bond along with the basal plan. Due to the external bias carrier conduction occur in this case is similar to the case of a planer npn or pnp transistor structure. The current flow is now confined and needs less amount of external energy in the case of these few layers bounded by covalent bond as the less amount of resistance faced by the carriers. As we can observe in the optical and SEM images, $\mathrm{ZrS}_{3}$ crystal has multilayer structure in which number of layers are stacked one upon the other and remain bonded with each other by weak van der Wall's bond. This kind of bonding needed large amount of external energy when carriers have to flow perpendicular to the basal plane i.e. anti-symmetric contact configuration. In this case all the layers of the crystal would take part in carrier transportation along the anti-symmetric configuration. This time carriers would face more resistance compared to the case of symmetric configuration which leads to the fall in the photocurrent.

The detector parameters such as responsivity $\left(R_{\lambda}\right)$, specific detectivity $(D)$, sensitivity $(S)$ and external quantum efficiency (EQE\%) are evaluated for $\mathrm{ZrS}_{3}$ using the following equations. 


$$
\begin{aligned}
& R_{\lambda}=\frac{I_{\text {ph }}}{P S} \\
& D=\frac{R_{\lambda} S^{1 / 2}}{\left(2 e I_{\text {dark }}\right)^{1 / 2}} \\
& S=\frac{I_{\text {ph }}}{I_{\text {dark }}} \\
& E Q E(\%)=\frac{h c R_{\lambda}}{e \lambda}
\end{aligned}
$$

Here, $I_{\mathrm{ph}}\left(I_{\mathrm{ph}}=I_{\mathrm{ill}}-I_{\text {dark }}, I_{\mathrm{ill}}=\right.$ current under illuminated condition and $I_{\text {dark }}=$ current under dark condition $)$ is the photocurrent, $\mathrm{P}$ is the illumination intensity, $\mathrm{S}$ is the effective area of the photodetector, $\mathrm{h}$ is Planck's constant, $c$ is the speed of light in vacuum, $\lambda$ is the wavelength of incident radiation, $e$ is the elementary electronic charge $(1.61019 \mathrm{C})$ and $\mathrm{I}_{\text {dark }}$ is dark current.

The l-t characteristics of the device was carried using same sources for both contact configurations at $1 \mathrm{~V}$ bias voltage which is shown in Fig. $\mathbf{4}$ (b) and (d). For both contact configurations, the current rises with illumination of light. The highest photocurrent was noted $245.7 \mathrm{nA}$ for symmetric contact and $33.54 \mathrm{nA}$ for anti-symmetric contact in the blue $(470 \mathrm{~nm})$ light illumination. This photocurrent reduced to $166.22 \mathrm{nA}$ for symmetric and $19.33 \mathrm{nA}$ anti-symmetric contact for the red $(670 \mathrm{~nm})$ light illumination.

The similar decrements are also observed in the detector parameters shown in Fig. 5. The graphs of the detector parameter i. e. photocurrent, sensitivity, responsivity, detectivity and EQE (\%) against wavelength are plotted. The highest responsivity and detectivity of $81.7 \mu \mathrm{A} / \mathrm{W}$ and $3.56 \times 10^{07}$ Jones were achieved for blue $(470 \mathrm{~nm})$ colour light respectively. It can be noted clearly from the scales of the all plots that the device shows significant anisotropy and with the increment of incident light wavelength, all the detector parameters are getting decrease for both types of contact configurations. So, the $\mathrm{ZrS}_{3}$ material in bulk crystal form possess good ability to be used in the preparation of the photo sensing device having anisotropic behaviour.

\section{Conclusion}

In summary, the $\mathrm{ZrS}_{3}$ single crystals are successfully synthesized by direct vapour transport technique. The EDAX characterization confirms that no impurity is found in the grown material and the mapping depicts the uniform distribution of the all-constituent elements. The optical images and SEM images shows that the grown material has layered kind of growth occurred which leads to the uniqueness in the behaviour of many detector parameters. From the study of current-voltage (I-V) and current-time (I-t) characteristics, it is clear that the $\mathrm{ZrS}_{3}$ material possess strong anisotropy as the device showed good performance for contact configuration made along the basal plane ( to c-axis) compared to the contact 
configuration made perpendicular to the basal plane ( to c-axis). Due to this uniqueness the material has good candidature for its application in the field of optoelectronic device applications.

\section{Declarations}

\section{Acknowledgment}

A.P. acknowledges the Government of Gujarat for providing financial support under the SHODH funding scheme (Ref No: 201901640001).

\section{References}

1. Bhimanapati, G. R. et al. Recent Advances in Two-Dimensional Materials beyond Graphene. ACS Nano9, 11509-11539 (2015).

2. Wang, Q. H., Kalantar-Zadeh, K., Kis, A., Coleman, J. N. \& Strano, M. S. Electronics and optoelectronics of two-dimensional transition metal dichalcogenides. Nat. Nanotechnol.7, 699-712 (2012).

3. Akinwande, D., Petrone, N. \& Hone, J. Two-dimensional flexible nanoelectronics. Nat. Commun.5, 112 (2014).

4. Dai, J., Li, M. \& Zeng, X. C. Group IVB transition metal trichalcogenides: a new class of 2D layered materials beyond graphene. Wiley Interdiscip. Rev. Comput. Mol. Sci.6, 211-222 (2016).

5. Srivastava, S. K. \& Avasthi, B. N. Preparation, structure and properties of transition metal trichalcogenides. J. Mater. Sci.27, 3693-3705 (1992).

6. Gorlova, I. G., Pokrovskii, V. Y., Zybtsev, S. G., Titov, A. N. \& Timofeev, V. N. Features of the conductivity of the quasi-one-dimensional compound TiS 3. J. Exp. Theor. Phys.111, 298-303 (2010).

7. Jin, Y., Li, X. \& Yang, J. Single layer of MX3 ( $M=T i, Z r ; X=S$, Se, Te): A new platform for nanoelectronics and optics. Phys. Chem. Chem. Phys.17, 18665-18669 (2015).

8. S.Furuseth, Brattas, L. \& A.Kjekshus. Acta_Vol_29a_P0623-0631.Pdf. vol. 29 623-631 (1975).

9. Galliardt, D. W., Nieveen, W. R. \& Kirby, R. D. Lattice properties of the linear chain compound TiS3. Solid State Commun.34, 37-39 (1980).

10. Grayfer, E. D., Pazhetnov, E. M., Kozlova, M. N., Artemkina, S. B. \& Fedorov, V. E. Anionic Redox Chemistry in Polysulfide Electrode Materials for Rechargeable Batteries. ChemSusChem10, 48054811 (2017).

11. Wu, J., Wang, D., Liu, H., Lau, W. M. \& Liu, L. M. An ab initio study of TiS3: A promising electrode material for rechargeable Li and Na ion batteries. RSC Adv.5, 21455-21463 (2015).

12. Giagloglou, K. et al. Zirconium Trisulfide as a Promising Cathode Material for Li Primary Thermal Batteries. J. Electrochem. Soc.163, A3126-A3130 (2016).

13. Manuscript, A. INORGANIC CHEMISTRY. (2014) doi:10.1039/C4QI00127C.

14. Pumera, M. TaS 3 Nano fi bers: Layered Trichalcogenide for High-Performance Electronic and Sensing Devices. (2018) doi:10.1021/acsnano.7b06853. 
15. Ait-Ouali, A. \& Jandl, S. Photoluminescence study of the one-dimensional material and its solid soluti. Phys. Rev. B - Condens. Matter Mater. Phys.53, 9852-9858 (1996).

16. Wang, X. et al. Highly Polarized Photoelectrical Response in vdW ZrS3 Nanoribbons. Adv. Electron. Mater.5, 1-8 (2019).

17. Tao, Y. R., Wu, X. C. \& Xiong, W. W. Flexible visible-light photodetectors with broad photoresponse based on ZrS3 nanobelt films. Smal/10, 4905-4911 (2014).

18. Tao, Y. R., Wu, J. J. \& Wu, X. C. Enhanced ultraviolet-visible light responses of phototransistors based on single and a few ZrS3 nanobelts. Nanoscale7, 14292-14298 (2015).

19. Chauhan, P. et al. Tunable and anisotropic photoresponse of layered Re0.2Sn0.8Se2 ternary alloy. Sol. Energy Mater. Sol. Cells200, 109936 (2019).

20. Wu, K. et al. Unusual lattice vibration characteristics in whiskers of the pseudo-one-dimensional titanium trisulfide TiS 3. 1-7 (2016) doi:10.1038/ncomms12952.

21. Talib, M., Tabassum, R., Sa, S. \& Mishra, P. Improvements in the Performance of a Visible - NIR Photodetector Using Horizontally Aligned TiS 3 Nanoribbons. (2019) doi:10.1021/acsomega.8b03067.

22. Levy, F. \& Berger, H. Single crystals of transition metal trichalcogenides f. 61, 61-68 (1983).

23. Dixit, V. et al. Growth, characterization and photoconduction properties of Sb0.1Mo0.9Se2 single crystals grown by DVT technique. Mater. Sci. Semicond. Process.88, 1-9 (2018).

24. Jethwa, V. P. et al. Temperature-dependent vibrational properties of DVT grown orthorhombic SnS single crystals and their application as a self-powered photodetector. Appl. Surf. Sci.531, 147406 (2020).

25. Zankat, C. K. et al. Materials Science in Semiconductor Processing Promoting photoresponse of resistive detector based on V 0 . 15 Sn 0 . 85 Se 2 ternary alloy. Mater. Sci. Semicond. Process.91, 383-386 (2019).

26. Guo, R., Wang, X., Kuang, Y. \& Huang, B. First-principles study of anisotropic thermoelectric transport properties of IV-VI semiconductor compounds SnSe and SnS. Phys. Rev. B - Condens. Matter Mater. Phys.92, 1-13 (2015).

27. Ho, C. H., Huang, Y. S. \& Tiong, K. K. In-plane anisotropy of the optical and electrical properties of ReS2 and ReSe2 layered crystals. J. Alloys Compd.317-318, 222-226 (2001).

28. Desai, S. C. et al. Thermoelectric Power Measurements of Zirconium Sulphoselenide Single Crystals. Nanotechnology19, 095507 (2008).

\section{Figures}




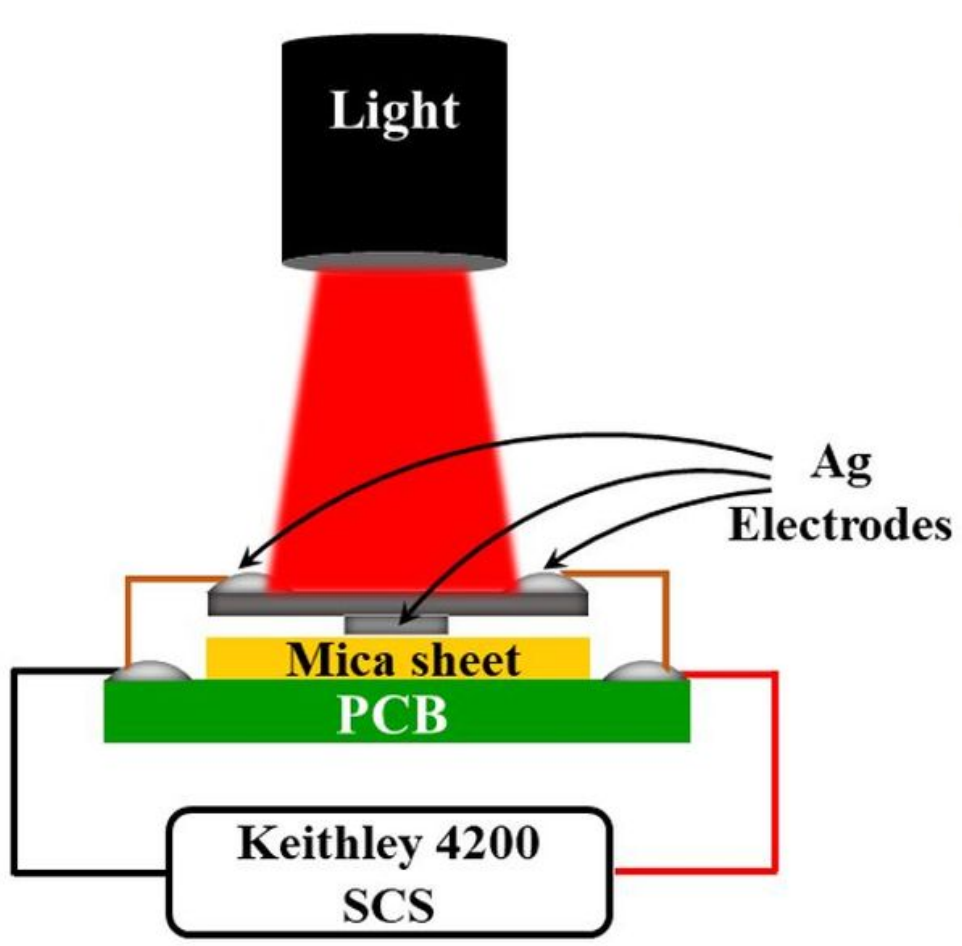

Conduction along the basal plane

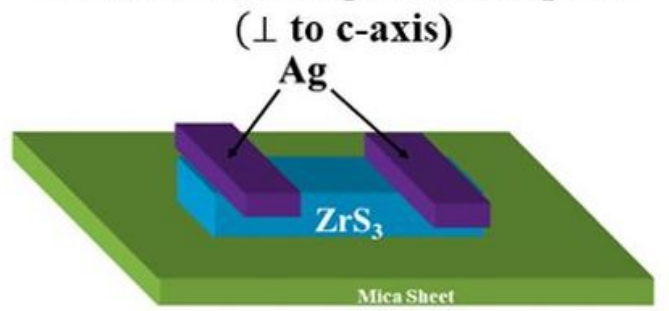

Conduction perpendicular to the basal plane (II to c-axis)

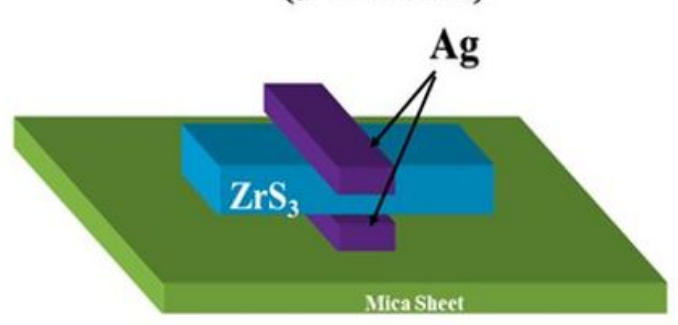

Figure 1

Schematic diagram of detector with two types of contact configurations
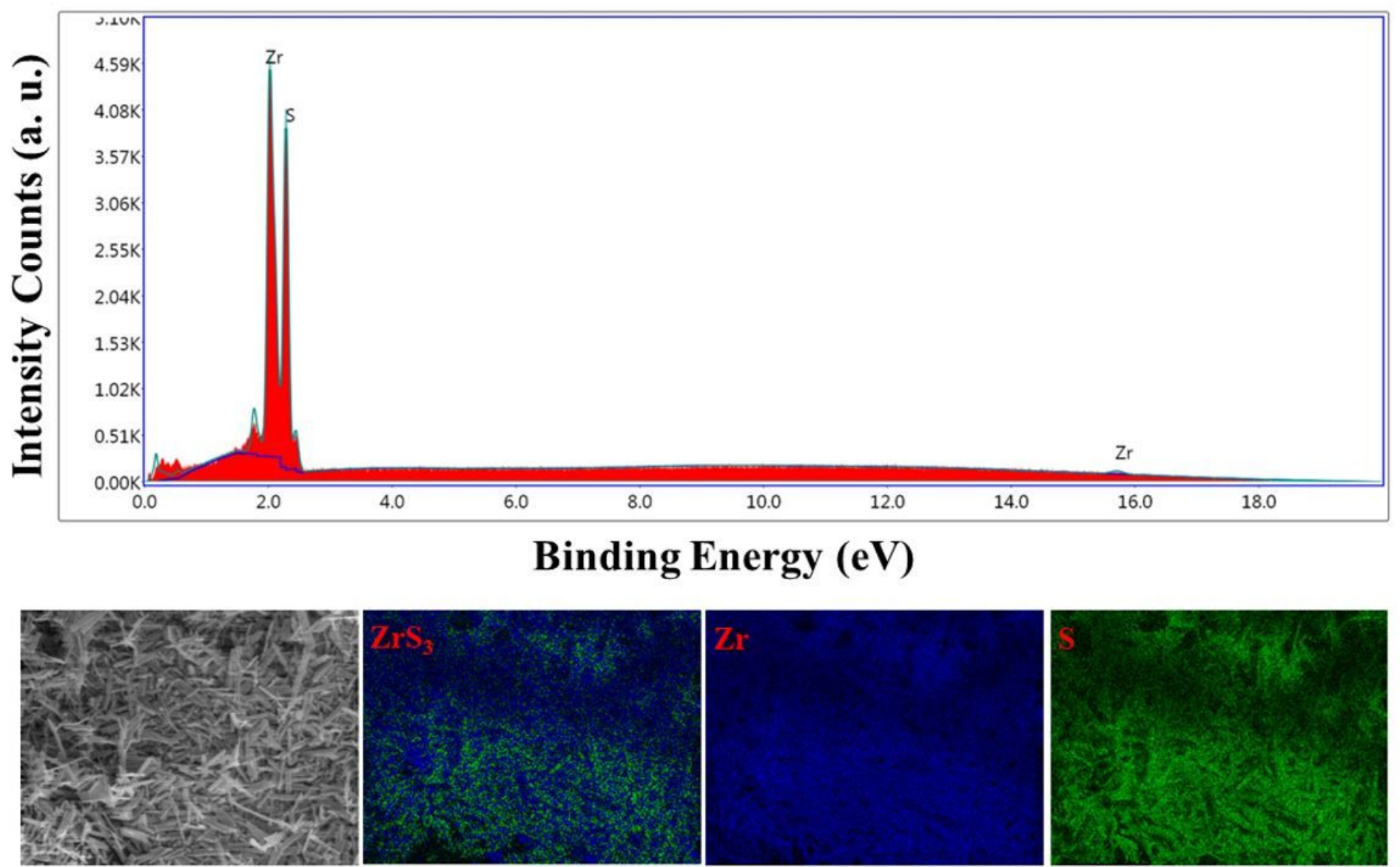


\section{Figure 2}

Elemental spectrum for ZrS3 as grown compound, the exposed area of the material for elemental mapping and uniform distribution of all the elements.
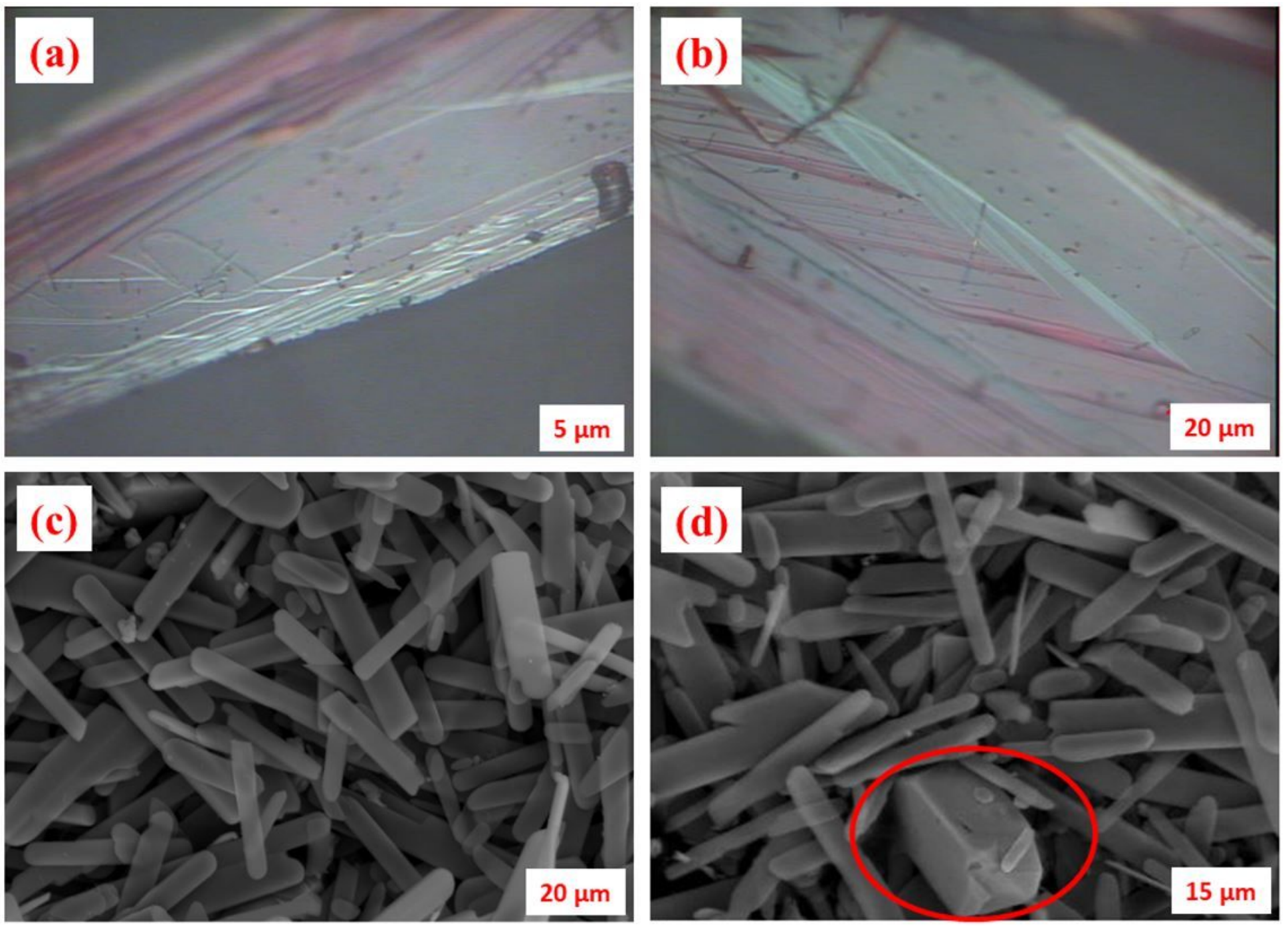

Figure 3

(a), (b) Optical microscopic image of ZrS3 crystal and (c), (d) Scanning electron microscopy of as grown compound of ZrS3. 

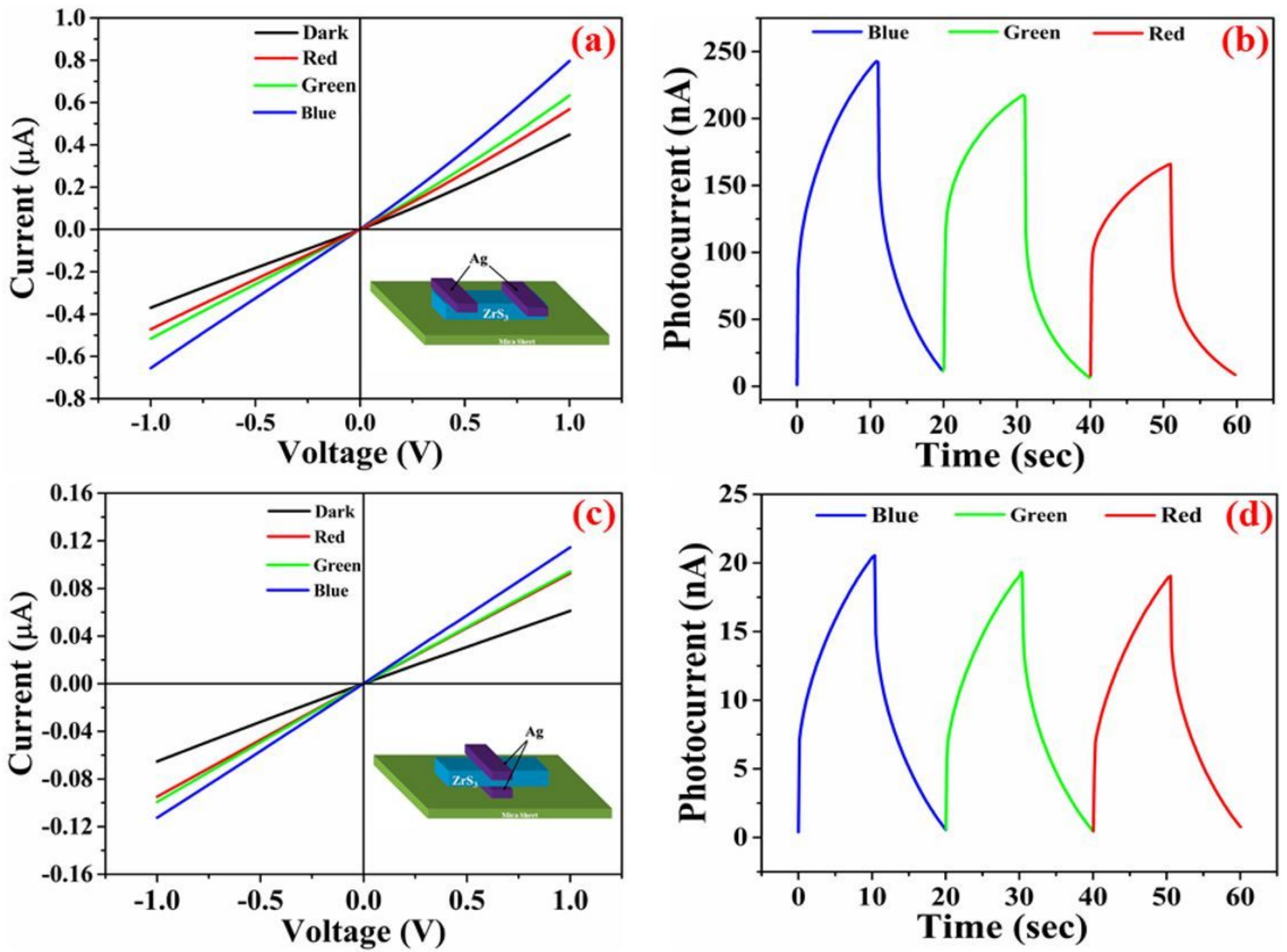

Figure 4

(a) and (c) the dark and illuminated currents of ZrS3 crystal for contacts along the basal plane and contacts parallel to c-axis, respectively. (b) and (d) Photo-response of ZrS3 crystal under different illuminating wavelengths for contacts along the basal plane and contacts parallel to c-axis, respectively. 

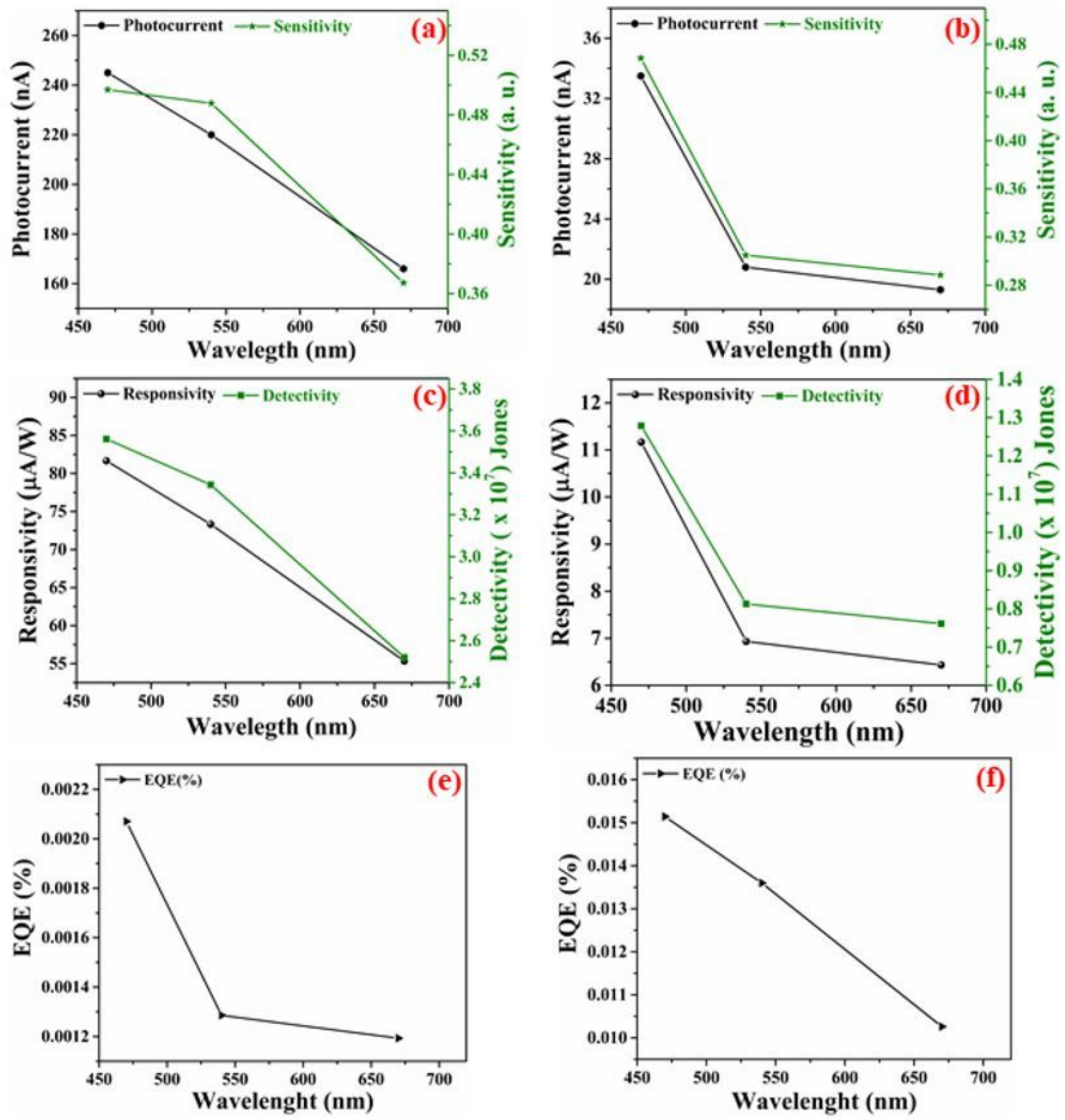

Figure 5

Detector parameters $(a, c, d)$ for contacts along the basal plane and $(b, d, f)$ for contacts perpendicular to the basal plane. 